\title{
Band 24, Heft 4, August 2012
}

Editorial

192 Integrative Schmerztherapie

Müller-Hübenthal, B. (Richterswil)

Im Fokus: Schmerztherapie

194 Schmerzen sind etwas Subjektives

197 Expertengespräch: Schmerztherapie

201 Mit Nadeln gegen Kopfschmerzen

Aus der Praxis für die Praxis

202 Schmerzen bei Behinderung: Mehr als doppelte Last! Ganzheitliche Behandlungsansätze in der Schmerztherapie Spreyermann, R. (Basel)

206 Hypnose als Teil der integrativen Diagnostik und Therapie kraniomandibulärer Dysfunktionen

Besimo, C.E. (Brunnen)

212 Ganzheitsmedizin aktuell

Fortbildung

214 Genussmittel und Ganzheitsmedizin (4): Kolanuss

Jeannin, J.-M. (Basel)

216 Die Kollegiale Instanz für Komplementärmedizin KIKOM als eines der führenden Forschungszentren für Komplementärmedizin Ausfeld-Hafter, B. (Bern)

218 Fachsymposium Gesundheit «Integrative Medizin und Pflege mehr als ein Trend» in St. Gallen

Roth Sigrist, M.; Schoop, B.; Mösli, N. (St. Gallen)
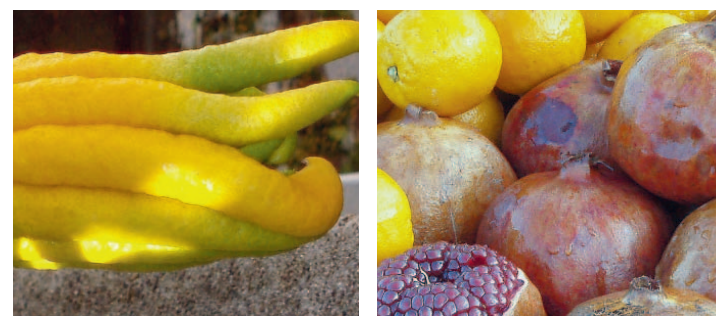

(c) Copyright by Jean-Michel Jeannin, Base

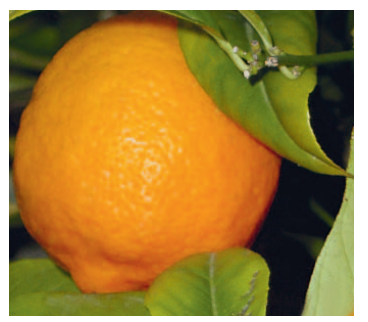

Schon seit sehr langer Zeit baut der Mensch Obst an und veredelt es. Obst ist eine reiche Quelle von Vitaminen, ätherischen Ölen und Antioxidantien. Der Verzehr von Obst in verschiedenster Form hilft, die Gesundheit zu erhalten, und bietet ein einzigartiges sinnliches Erlebnis. Die Reihe meist besonderer Früchte zeigt von links nach rechts: Zitronatszitrone (Citrus medica var. sarcodactylis) (Botanischer Garten Basel, Mai 2011), Granatäpfel (Punica granatum) und Zitronen (Citrus limon) auf einem Markt (Istanbul, Juni 2011) sowie Lemandrinen (Citrus x limonium Osbeck) (Botanischer Garten Basel, Oktober 2010).

\section{KARGER}




\section{Übersichtsarbeit (Peer Review)}

221 Molekulare Stoffwechselsensorik bei durch Adipositas induzierter Entzündung

Molecular Metabolic Sensors in Obesity-Induced Inflammation

Sensorique moléculaire du métabolisme en cas d'inflammation induite par l'obésité Eisenstecken, E. (Kufstein); Gostner, J.H.; Fuchs, D.; Überall, F. (Innsbruck)

Essay (Peer Review)

233 Thure von Uexkülls semiotisches Modell des Menschen als Grundlage für eine moderne Theorie der Homöopathie

Thure von Uexküll's Semiotic Model of Man as a Basis for a New Theory of Homeopathy Modèle sémiotique de l'homme par Thure von Uexküll comme base d'une théorie moderne de l'homéopathie

Schmidt, J.M. (München)

242 Ist die Aristolochiasäure in der Asarum-Pflanze tatsächlich kanzerogen? Die wichtige Bedeutung epidemiologischer Effektmodifikation mit einem klinischen Fallbeispiel

Is the Carcinogenic Effect of Aristolochic Acid in Asarum Plants Well-Established? The Important Role of Effect Modification in Epidemiologic Analysis Including a Clinical Case Study

L'acide aristolochique que contient la plante appelée «asaret» est-il bel et bien cancérigène? L'importance déterminante de la modification de l'effet sur le plan épidémiologique illustrée par un exemple clinique

Schmidt, J.G. (Einsiedeln); Bühlmann, S. (Schlieren); Shao, Y. (Einsiedeln)

249 Bildungsmarkt

250 Buchbesprechungen

Mitteilungen UNION

252 Berufsbilder Komplementärtherapeut/in und Naturheilpraktiker/in Imfeld, S. (Kägiswil)

253 Tagungen und Kongresse

248 Impressum
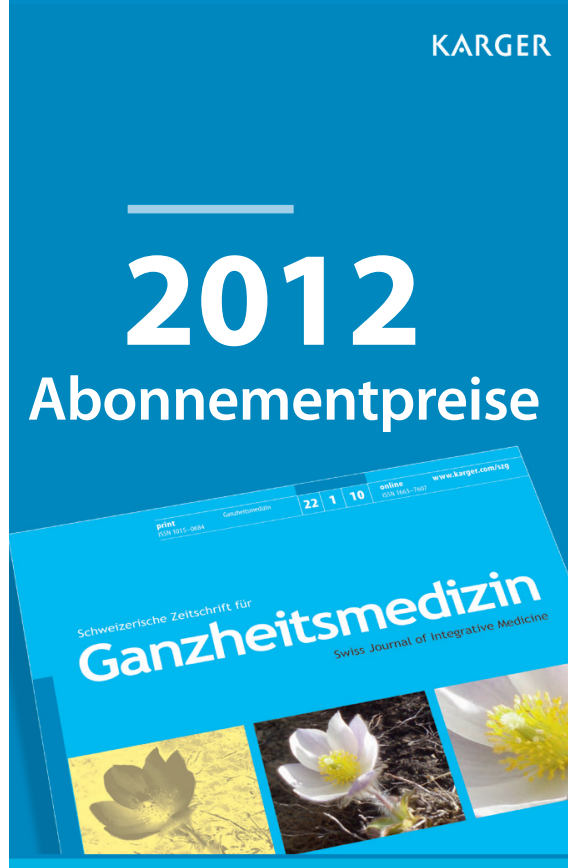

Band 24 mit 6 Heften

- Print EUR 58,- / CHF 75,-

- Online EUR 58,- / CHF 75,-

- Kombi EUR 72,- / CHF 94,-

Versandkosten für Print und

Kombi-Abonnement

EUR 20,- / CHF 30,-

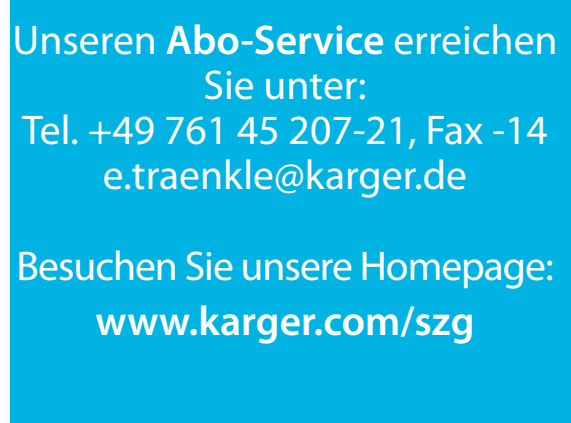

